\title{
Editorial
}

\section{Nanostructured Thin Films and Coatings}

\author{
Ping Xiao' and Robert Dorey ${ }^{2}$ \\ ${ }^{1}$ School of Materials, University of Manchester, Grosvenor Street, Manchester M1 7HS, UK \\ ${ }^{2}$ School of Applied Sciences, Microsystems and Nanotechnology Centre, Cranfield University, \\ Cranfield, Bedfordshire MK43 OAL, UK
}

Correspondence should be addressed to Ping Xiao,ping.xiao@manchester.ac.uk

Received 1 April 2008; Accepted 1 April 2008

Copyright (c) 2008 P. Xiao and R. Dorey. This is an open access article distributed under the Creative Commons Attribution License, which permits unrestricted use, distribution, and reproduction in any medium, provided the original work is properly cited.

The field of films and coating continues to develop with new materials, processing, and applications being envisaged. This special edition of Journal of Nanomaterials examines some of the latest developments in nanostructured thin films and coatings. Nanostructured thin films and coatings possess properties different to homogenous materials due to the deliberate engineering of nanoscale features into the structure. The seven papers presented in this special edition examine the challenges faced in fabricating nanoscale systems and composites, as well as the electrical and light interactions that occur with such small-scale systems

In the first paper, glass plate-supported nanostructure $\mathrm{ZnO}$ thin films were deposited by sol-gel spin coatings. The $\mathrm{ZnO}$ thin films are transparent ca $80-90 \%$ in visible range, and have absorption edge at about $370 \mathrm{~nm}$. The $\mathrm{c}$-axis orientation improves and the grain size increases, which was indicated by an increase in intensity of the (002) peak at 34.40 in XRD corresponding to the hexagonal $\mathrm{ZnO}$ crystal. The photocatalytic degradation of X6G, an anionic monoazo dye, in aqueous solutions was investigated, and the effects of some operational parameters such as the number of layers and reusability of $\mathrm{ZnO}$ nanostructure thin film were examined. The results showed that the five-layer coated glass surfaces have a very high photocatalytic performance.

In the second paper, the microstructure and photocatalytic performance of titania coatings obtained by different thermal spray techniques were investigated: atmospheric plasma spraying (APS), suspension plasma spraying (SPS) alternative plasma spray technique using liquid feedstock material and the high-velocity oxygen fuel spray process (HVOF). Various titania powders were used as feedstock materials for spraying. Different $\mathrm{TiO}_{2}$ nanopowder suspensions were carried out to study the influence of the solvent nature on the coating characteristics. The photocatalytic efficiency of the elaborated samples was tested in an environmental test chamber setup and evaluated from the gaseous nitrogen oxide (NO, NOx) removal.

In the third paper, the structural, optical, and conductivity properties of silicon film deposited on cerium dioxide buffer layer were studied. The data of Raman scattering measurements, scanning electron microscopic experiments, and electrophysical measurements were used. The temperature spatial distribution and cerium dioxide phase conditions play a great role in silicon crystallization. Applied external electric field was used to destroy the crystal structure order in silicon. Crystal phase of silicon film was restored by annealing the samples. The field destruction of crystal phase and its restoration after annealing were investigated by Raman spectroscopy.

In the fourth paper, a study was concentrated on preparation and structural characterization of granular $\mathrm{Ag}-\mathrm{ZrO}_{2}$, $\mathrm{Co}-\mathrm{ZrO}_{2}$, and $\mathrm{Au}-\mathrm{ZrO}_{2}$ thin films grown by pulsed laser deposition (PLD) in a wide range of volume fraction $\mathrm{x}$ of metal $(0.08<\mathrm{xAg}<0.28,0.06<\mathrm{xCo}<0.40$, and $0.08<\mathrm{xAu}$ $<0.55)$. High-resolution transmission electron microscopy (HRTEM) showed regular distribution of spherical $\mathrm{Au}, \mathrm{Co}$, and $\mathrm{Au}$ nanoparticles having very sharp interfaces with the amorphous matrix. The structural results are compared aiming to stress the effect of the actual microstructure on the percolation threshold. Two different mechanisms of particle growing as a function of the metal content are evidenced: nucleation and particle coalescence, with 
their relative significance depending strongly on the type of metal, giving rise to very different values of the percolation threshold $(\mathrm{xc}(\mathrm{Ag}) \sim 0.28, \mathrm{xc}(\mathrm{Co}) \sim 0.35$, and $\mathrm{xc}(\mathrm{Au}) \sim 0.55)$.

In the fifth paper, inorganic thin films (hydroxide, oxide, and phosphate materials) that are textured on a submicron scale have been prepared from aqueous metal salt solutions at room temperature using vapor-diffusion catalysis. This generic synthesis approach mimics the essential advantages of the catalytic, and structure-directing mechanisms observed for the formation of silica skeletons of marine sponges. Chemical composition, crystallinity, and the three-dimensional morphology of thus prepared films are extremely sensitive to changes in the synthesis conditions, such as concentrations, reaction times and the presence and nature of substrate materials. Focusing on different materials systems, the reaction mechanism for the formation of these thin films and the influence of different reaction parameters on the product are explained.

In the sixth paper, chromium metal-induced nanocrystallization of amorphous silicon (a-Si) thin films is reported. The nanocrystalline nature of these films is confirmed from X-ray diffraction and Raman spectroscopy. Significantly, the deconvolution of Raman spectra reveals that the thin films were crystallized in a mixed phase of cubic diamond and wurzite structure as evidenced by the lines at 512 and $496 \mathrm{~cm}^{-1}$, respectively. The crystallite sizes were between 4 and $8 \mathrm{~nm}$. Optical properties of the crystallized silicon, derived from spectral transmittance curves, revealed high transmission in the region above the band gap. Optical band gap varied between 1.3 and $2.0 \mathrm{eV}$, depending on the nature of crystallinity of these films, and remained unaltered with an increase in Cr addition from 5 to $30 \%$. This signifies that the electronic structure of the nanocrystalline silicon films is not affected considerably inspite of the presence of metal silicides and the process of crystallization.

In the final paper, the thermomechanical stresses acting between a nanotube and fullerenes encapsulated on it are computed. After a general formulation, based on elasticity, the analysis suggests that a thermal control could be used to produce smart fullerenes at nanotube systems, for example, as two-stage nanovectors for drug delivery.

Ping Xiao Robert Dorey 

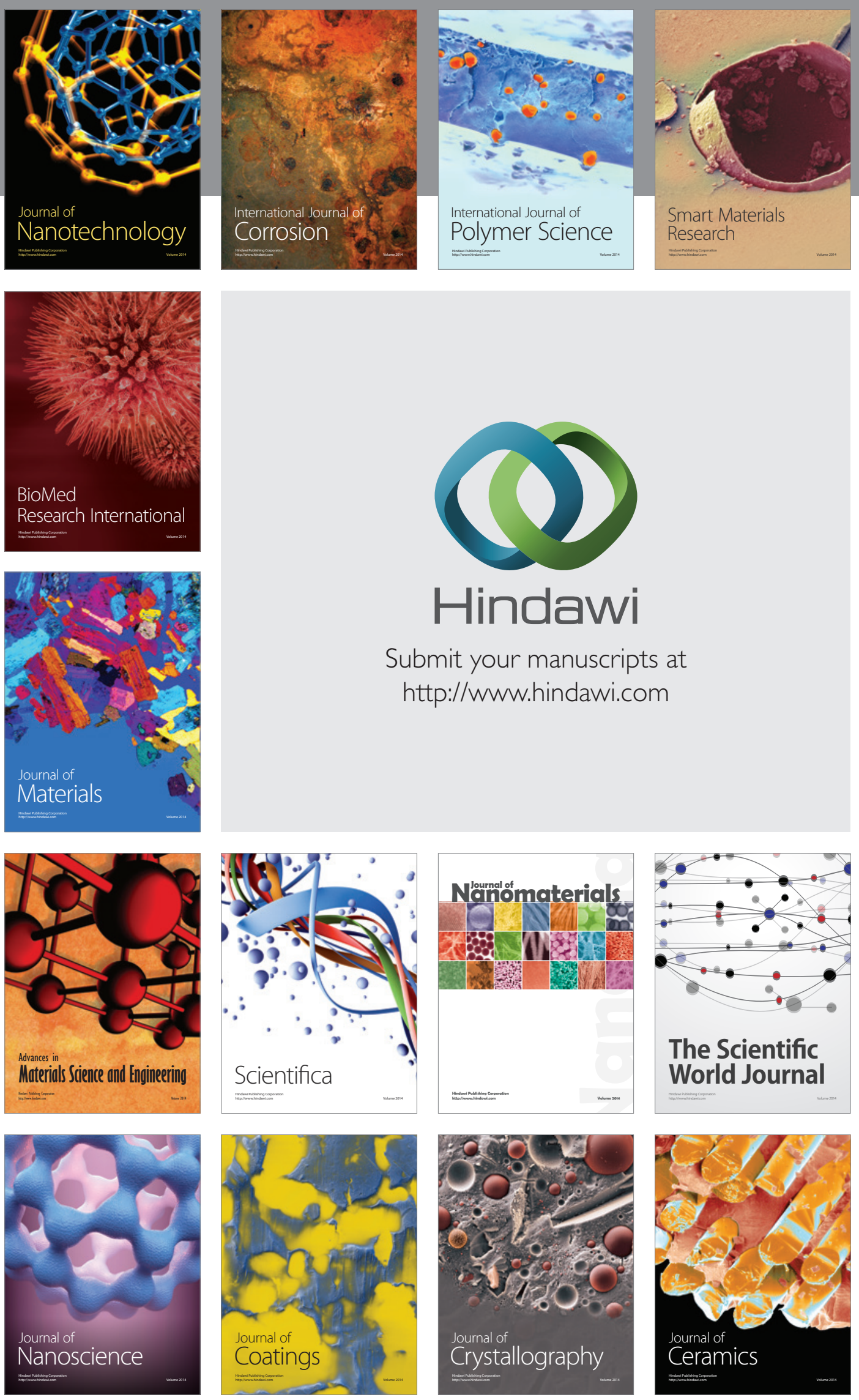

The Scientific World Journal

Submit your manuscripts at

http://www.hindawi.com

\section{World Journal}

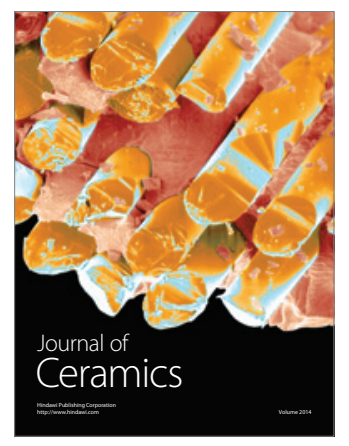

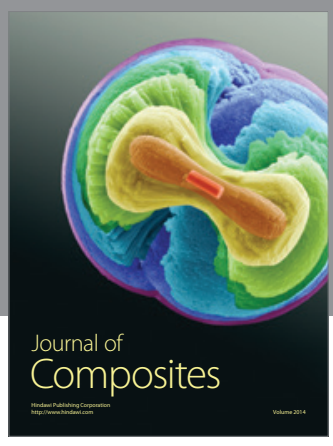
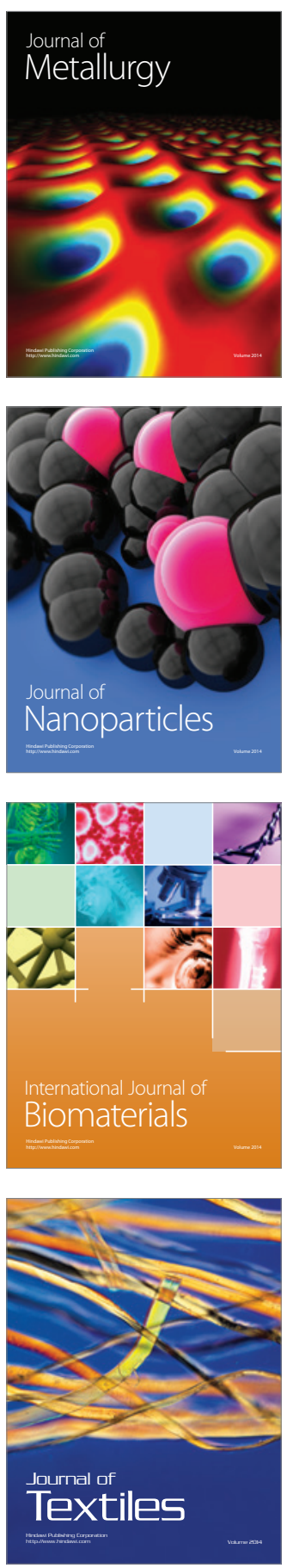